'Unidad de Psiquiatría de Enlace Hospital Santiago Oriente Dr. Luis Tisné B. Santiago, Chile. ${ }^{2}$ Centro Comunitario de Salud Mental (COSAM) Peñalolén. Santiago, Chile.

${ }^{3}$ Servicio Médico Legal Metropolitano. Universidad Andrés Bello. Santiago, Chile.

${ }^{4}$ Programa de Acogida y Acompañamiento Psicosocial Hospital Santiago Oriente Dr. Luis Tisné B. Santiago, Chile.

aDoctor en Psiquiatría Forense.

Trabajo no recibió financiamiento. Los autores declaran no tener conflictos de interés.

Correspondencia a: Pablo Muñoz El Trovador 4280 Oficina 603 Las Condes. Santiago. pablomunozparra@gmail.com

\section{Dilemas clínicos en la constitución de la tercera causal de la interrupción voluntaria del embarazo}

\author{
PABLO MUÑOZ ${ }^{1,2}$, JAVIERA PARRINI ${ }^{1}$, \\ RODRIGO DRESDNER ${ }^{3, a}$, MIRTHA JIMÉNEZ ${ }^{4}$
}

Since 2017, women in Chile are allowed to interrupt voluntarily a pregnancy on three grounds: 1) When a woman's life is at risk due to the pregnancy, 2) When there are fetal anomalies incompatible with life, or 3) When pregnancy is result of rape. Women who qualify for any of these three pregnancy interruption requirements are entitled to a psychosocial accompaniment program to promote an integrative approach. In this article we will discuss the role of the psychosocial team in cases of rape resulting pregnancy. Specifically, the clinical and ethical dilemmas posed by the need to certify the rape in a general hospital such as difficulties in assessment of the story's plausibility, clinician's dual role and the limits to confidentiality, are discussed.

(Rev Med Chile 2021; 149: 758-764)

Key words: Abortion, Induced; Abortion, Legal; Pregnancy, Unwanted; Psychological Trauma; Rape.

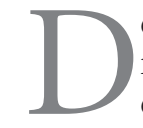
esde 1989, Chile tenía una de las legislaciones más restrictivas en relación al aborto en el mundo, ya que no lo permitía bajo ninguna circunstancia ${ }^{1}$. Esto cambia en septiembre de 2017, cuando se promulga la ley No 21.030 que regula la despenalización de la Interrupción Voluntaria del Embarazo (IVE) en tres causales: 1) Peligro de la vida de la mujer embarazada, 2) Inviabilidad fetal de carácter letal y 3 ) Embarazo producto de una violación ${ }^{2}$ (Tabla 1 ).

Para la implementación de la ley se han constituido equipos multidisciplinarios (psiquiatra, psicólogo, trabajadora social, obstetra) que realizan el procedimiento de la IVE con el objetivo de asegurar "el acceso, oportunidad, calidad y seguridad de la atención, resguardando la continuidad del proceso y relevando el acompañamiento psicológico y social, evitando la revictimización, en un ambiente de respeto y confidencialidad"3.
Se entiende la interrupción del embarazo como: "la intervención instrumental y/o medicamentosa para poner fin a un embarazo". Por lo tanto, si dicha intervención se realiza antes de la viabilidad fetal (establecida hasta las 22 semanas de gestación) se considera un aborto. Pero si se realiza después de este período, constituye una inducción de un parto prematuro ${ }^{4,5}$.

El debate sobre el aborto se exacerba frecuentemente por sesgos, juicios de valor, ideologías, presiones políticas, doctrinarias y religiosas ${ }^{4}$ y suele darse entre posturas antagónicas que representan visiones irreconciliables del problema ${ }^{6}$, sin embargo, éstas escapan al objetivo de este artículo.

Considerando que la ley ya se encuentra vigente, nos propusimos revisar el rol del equipo psicosocial en la tercera causal de la Ley IVE (embarazo por violación), porque involucra - por definiciónuna de las formas más severas de trauma psíquico 


\section{Tabla 1. Causales de Interrupción voluntaria del embarazo}

Causal 1: Peligro de vida para la mujer

En la primera causal se autoriza la interrupción del embarazo cuando la mujer se encuentra en riesgo vital, de modo que la interrupción del embarazo evite un peligro para su vida.

En esta causal no hay un plazo para la interrupción, se puede realizar a lo largo del embarazo.

\section{Causal 2: Inviabilidad fetal de carácter letal}

Cuando un embrión o feto padezca una patología congénita adquirida o genética, incompatible con la vida extrauterina independiente, en todo caso de carácter letal.

Como el embrión o feto no logrará sobrevivir fuera del útero, la interrupción de estos embarazos no está supeditada a plazos, sino a la decisión de la mujer.

\section{Causal 3: Embarazo por violación}

La ley IVE autoriza la interrupción cuando el embarazo es resultado de una violación, siempre que no hayan transcurrido más de 12 semanas de gestación. En el caso de las menores de 14 años, la interrupción del embarazo podrá realizarse hasta las 14 semanas de gestación.

Para acreditar la concurrencia de la causal la ley exige que un equipo de salud, especialmente conformado para estos efectos, confirme la concurrencia de los hechos que lo constituyen y la edad gestacional.

y, además, porque compete al equipo psicosocial establecer la "constitución de la causal", instancia que cuenta con algunas particularidades desde el punto de vista clínico, ético y médico-legal, que desarrollaremos a lo largo del texto.

\section{Rol del equipo psicosocial y acompañamiento terapéutico}

La normativa ministerial hace énfasis en un abordaje integral de la IVE en el contexto de un modelo de atención multidisciplinario biopsicosocial y dispone que las mujeres que presenten algunas de las causales de IVE, independiente de su decisión de continuar o interrumpir su embarazo, tienen derecho a un programa de acompañamiento, tanto en el proceso de discernimiento como durante el período siguiente a la toma de decisión.

El acompañamiento psicosocial es definido en la Orientación Técnica como "un proceso que desarrolla el equipo interdisciplinario de salud, en articulación con el intersector, a través del cual acoge y acompaña a la adolescente o mujer embarazada que se acoge a la Ley"7.

Es siempre voluntario y respetuoso de la libre decisión de la mujer y no puede ser impuesto ni requisito para optar a la IVE. Además, puede ser solicitado por la paciente en cualquier momento del proceso.

Para el acompañamiento psicosocial en todas las causales, la normativa estructura el proceso en fases: acogida, acompañamiento en la decisión y continuidad del acompañamiento (seguimiento). Sin embargo, es importante destacar que en la tercera causal, además de los roles y consideraciones psicológicas de estas tres fases para la interrupción en un embarazo por violación, el equipo psicosocial también debe confirmar la concurrencia de los hechos que constituyen la causal (Tabla 2).

Tabla 2. Rol del equipo psicosocial en las tres causales de la IVE

\begin{tabular}{|c|c|c|c|}
\hline & Causal 1 & Causal 2 & Causal 3 \\
\hline Acogida & + & + & + \\
\hline Acompañamiento en la decisión & + & + & + \\
\hline Continuidad del acompañamiento & + & + & + \\
\hline Constitución de la causal & & & + \\
\hline
\end{tabular}




\section{Dilemas clínicos en la constitución de causal}

En la ley se le exige al equipo psicosocial la evaluación de la concurrencia de la tercera causal: "En el caso del número 3) del inciso primero del artículo 119, un equipo de salud, especialmente conformado para estos efectos, confirmará la concurrencia de los hechos que lo constituyen y la edad gestacional, informando por escrito a la mujer o a su representante legal, según sea el caso, y al jefe del establecimiento hospitalario o clínica particular donde se solicita la interrupción"2.

Teniendo en cuenta que la tercera causal tiene plazos específicos establecidos por la ley, esta evaluación y la confirmación de los hechos que constituyen la causal exigen celeridad, debiendo entregar un informe escrito en un plazo de $24 \mathrm{~h}$.

\section{Análisis de la plausibilidad del relato}

La normativa describe que la concurrencia de la causal debe ser evaluada por los equipos de salud sobre la base de las siguientes consideraciones ${ }^{3}$ :

1. Análisis de la plausibilidad del relato.

2. Idoneidad de los hechos relatados para producir un embarazo.

3. Concordancia estimada entre la fecha de la violación relatada y la edad gestacional informada.

Con el relato de la experiencia traumática en el contexto de una entrevista clínica, en la mayoría de los casos, es posible determinar la idoneidad de los hechos para producir un embarazo y una concordancia estimada entre la fecha de la violación aportada por la paciente y la edad gestacional; sin embargo, el requerimiento de un análisis de la plausibilidad del relato es una tarea que ha resultado altamente compleja. Hasta ahora el único elemento objetivo para no constituir la causal es la discordancia entre la edad gestacional y la fecha probable de violación. En estos casos, se le ofrecen a la mujer o adolescente, las alternativas disponibles de apoyo para continuar con la gestación.

La norma indica que se debe distinguir entre la valoración que realiza el equipo de salud y el "peritaje para evaluación de veracidad del relato", a cargo de la instancia judicial pertinente. Es más, aclara que el equipo de salud no tiene competencias investigativas y no sustituye ni al Ministerio Público ni a los tribunales en la comprobación de la concurrencia del delito de violación ni en la determinación de sus autores. Sin embargo, en el mismo capítulo describe ciertos elementos en los antecedentes que el equipo psicosocial debiese considerar, pues respaldan la sospecha fundada de violación, tales como: características de la memoria y el recuerdo; el contexto en que se da la develación; correlato conductual y emocional frente a la experiencia traumática; actitud frente a la denuncia y momento del ciclo vital y etapa del desarrollo en que sucede la violación.

No obstante, en el ámbito médico legal, específicamente en la psiquiatría forense, hasta ahora técnicamente no ha sido posible desarrollar una metodología de examen que posibilite detectar o distinguir la verdad de la mentira en el relato de una persona adulta. Y de ahí que el Servicio Médico Legal en la Guía Normativa Técnica Pericial-que emite y actualiza regularmente al tenor del estado de arte pericial en el campo de salud mental- señala que "la evaluación del testimonio se realizará solo en personas mayores de 3 años y menores de 18 " 8 .

Asimismo, la Guía Normativa Técnica Pericial define peritaje como: "Proceso de examen, análisis y evaluación, efectuado por un perito de la ciencia, arte $\mathrm{u}$ oficio o requerido, que culmina con un documento escrito que contiene los resultados de ese proceso, como parte de una solicitud de los Órganos Jurisdiccionales o de Investigación"8. Esta definición tiene evidentes similitudes con el proceso que se lleva a cabo en la constitución de causal, ya que es la realización de un proceso de evaluación que culmina con un informe escrito respecto a los hechos relatados por la paciente, por lo que los límites entre estos dos procesos son poco claros.

Según entendemos, lo que se le solicita al equipo psicosocial en la constitución de causal evidencia una profunda contradicción: si bien la norma intenta distinguir la constitución de causal de un peritaje, exige, por otro lado, una evaluación exhaustiva del relato para discriminar si constituye o no una violación. Esto ya fue advertido por Bórquez et al. en el 2015, quienes consideraron altamente gravoso imponer al equipo de salud la carga de evaluar e informar la concurrencia de los hechos que constituyen la causal y, además, de gran dificultad acreditar adecuadamente su concurrencia 6 .

Otro dilema que surge es: ¿cuál es la conducta más adecuada cuando el clínico tiene "dudas 
respecto a la plausibilidad del relato"? En Chile no existe una claridad de cuál es la conducta que corresponde y probablemente en la práctica va a depender de las creencias, experiencias y la ética profesional. Algunos van a optar por dudar y tomar un rol más investigativo; otros, quizá, van a optar por no cuestionar y ante la duda constituir la causal. El conflicto que subyace a estas preguntas es que como clínicos tenemos que sopesar, en primera instancia, llevar a cabo un riguroso análisis de la plausibilidad del relato versus no revictimizar a una paciente que ha sufrido un trauma reciente.

Los países en Latinoamérica manejan distintos modelos de permiso para la causal de violación. Es posible distinguir aquellos que exigen requisitos específicos previos para la realización del aborto (denuncias o trámites judiciales), como Bolivia, Panamá, Colombia y Costa Rica, de aquellos que los excluyen expresamente o no los prevén, como Brasil o Argentina ${ }^{9-12}$. La ley IVE plantea una propuesta intermedia entre estos dos modelos: no tiene el requisito de una denuncia o trámite judicial previo; sin embargo, sí requiere una autorización del equipo psicosocial o "constitución de causal”.

A nuestro parecer la constitución de causal es una situación insoluble en la práctica clínica y obliga al equipo psicosocial a tomar una decisión y elaborar un informe que está poco sustentado científicamente y que se asemeja a un peritaje. No encontramos en la literatura internacional un ejemplo similar a la constitución de causal como requisito para un aborto.

A modo de ejemplo, la legislación argentina resuelve las dudas respecto a la plausibilidad del relato explicitando que el único requisito para acceder a una interrupción legal del embarazo (ILE) es que la mujer realice una declaración jurada donde afirme que el embarazo que cursa es producto de una violación ${ }^{13}$. En este sentido, la guía clínica de aborto no punible en Argentina cita el fallo de la Corte Suprema (CSJN), donde aclara que ante una duda sobre la veracidad de la violación es prioridad no negar bajo ninguna circunstancia el acceso al servicio de ILE $^{14}$. A pesar de que la CSJN advierte la posibilidad de configuración de "casos fabricados", considera que el riesgo derivado del irregular obrar de determinados individuos no puede ser nunca razón suficiente para imponer a las víctimas de delitos sexuales obstáculos que vulneren el goce efectivo de sus legítimos derechos o que se constituyen en riesgos para su salud ${ }^{14}$.

\section{Doble rol}

Por tanto, la constitución de causal nos va a plantear inevitablemente ciertos dilemas clínicos y éticos. Primero nos pone en un doble rol como clínicos ante estas pacientes, ya que por un lado somos garantes de certificar con nuestras competencias clínicas si el relato que escuchamos corresponde o no a una violación y, a pesar de que la normativa haga la distinción con un peritaje, vamos a estar en una posición de "juez" que decide si se constituye o no la causal. Pero al mismo tiempo y en la misma entrevista, vamos a estar conociendo y estableciendo un vínculo terapéutico con la paciente, pues somos nosotros quienes, en caso de que ella aceptara, llevaremos a cabo el acompañamiento psicológico. Es evidente el conflicto clínico: ¿usamos un paradigma de confianza o de duda?, ¿tomamos un rol inquisitivo o investigativo en nuestra entrevista o confiamos $a$ priori en lo que la paciente nos relata?

De hecho, en el ámbito médico-legal se busca evitar este doble rol por ser excluyente el rol médico de la función como testigo. De ahí que en el ámbito médico-legal no es aconsejable que los psiquiatras legistas oficien como tratantes de personas que, previamente, han peritado; $y$, a su vez, existe una franca incompatibilidad en que un médico oficie de perito respecto de un/una paciente.

En nuestro equipo, ante el conflicto descrito intentamos privilegiar el rol clínico, a través de la escucha activa, sin juicios ni confrontaciones para evitar la revictimización, sin dejar por esto de cumplir con las disposiciones exigidas en la ley.

\section{Confidencialidad "con reparos"}

En los principios del modelo de atención de la Norma Técnica se procura velar por el "respeto a la dignidad, privacidad y confidencialidad que requiere toda atención clínica"3. Establece que se le debe preguntar a la mujer solo aquello necesario para el diagnóstico y manejo clínico del problema de salud que presenta; sin embargo, para constituir la tercera causal -apegados a la norma- nos podríamos ver en la obligación de, efectivamente, preguntar aspectos de la historia y del relato de la violación que van más allá del manejo clínico del problema. 
Además, en la Norma se propone invitar a la víctima a conversar de manera abierta, informándole del compromiso del equipo de salud de resguardar la confidencialidad de su historia; pero, a la vez, le debemos informar que es necesario evaluar si se constituye o no la causal para interrupción del embarazo y que, además, los jefes de establecimientos hospitalarios o clínicas deben poner en conocimiento al Ministerio Público del delito de violación con la finalidad que se investigue de oficio al o los responsables. Por ende, al comenzar la entrevista la paciente ya está al tanto de nuestro doble rol.

Otro aspecto que podría influir en la percepción de la paciente respecto a la confidencialidad de su relato, es la toma obligatoria de una muestra de restos fetales que se guarda bajo medidas de seguridad hasta por un año, con el fin de establecer, en lo posible, la vinculación del agresor con la víctima y la identificación del agresor mediante la recuperación de $\mathrm{ADN}$.

Esto debe ser advertido de antemano, por lo que es probable que la paciente sepa que a pesar de nuestro compromiso de respetar su confidencialidad, ésta va a tener algunos "reparos". Es decir, aunque va a ser confidencial es necesario notificar al Ministerio Público y, además, guardar una muestra de restos fetales para un eventual peritaje.

Una eventual alternativa a la situación descrita sería que las muestras fetales fueran guardadas y el informe fuera emitido solo en los casos en los cuales la paciente decida efectivamente realizar la denuncia. De esa manera la decisión dependería de la usuaria y no tendría que ser impuesta por el equipo clínico.

\section{Discusión}

En este artículo hemos procurado describir en qué consiste la Ley IVE a nivel general y hemos querido profundizar, particularmente, en la tercera causal, dando énfasis al rol que cumple el equipo psicosocial, el cual en nuestra experiencia es fundamental en la atención de las pacientes que han sufrido un embarazo producto de una violación. Por estudios en otros países, sabemos que solo una de cada tres mujeres que ha sufrido una violación busca ayuda médica ${ }^{15}$, por lo que el aborto puede llevar a una mujer con un embarazo producto de la misma, por primera vez, a acceder a apoyo y cuidados luego de la agresión. Esto pone al equipo psicosocial en un lugar privilegiado para acoger empáticamente, intervenir y acompañar en esta sensible etapa a la víctima, sin embargo, que sea la misma dupla psicosocial quien, además, tenga la tarea de constituir la causal conlleva ciertas dificultades que hemos ido revisando a lo largo del artículo.

$\mathrm{Al}$ analizar el rol del equipo psicosocial en la tercera causal, surgen varias preguntas y cuestionamientos. ¿Corresponde al equipo psicosocial constituir tercera causal? ¿Puede el equipo psicosocial ofrecer realmente confidencialidad a la paciente cuando al mismo tiempo debe referirle que está en la obligación de comunicar los hechos al Ministerio Público? ¿Cuáles son las implicancias clínicas de que el mismo equipo que constituye causal sea el que realice el posterior acompañamiento?

Intentando responder algunas de las preguntas anteriores, pensamos que el requerimiento de que un profesional de la salud "confirme la concurrencia de hechos" -cualesquiera sean ellos- que no presenció, médico-legalmente y bajo todo punto de vista es cuestionable. En ese caso, la confirmación de ocurrencia de hechos delictuales es tarea y responsabilidad exclusiva del ente investigador penal. Creemos que a pesar de que la normativa intenta hacer una diferencia entre la constitución de tercera causal y un proceso pericial, hemos detectado que en la práctica existen varias similitudes, lo que lleva a una diferenciación difusa entre estos procesos. Considerando la difícil tarea que implica realizar un análisis de la plausibilidad del relato en una paciente y que, además, adolece de un alto margen de subjetividad de un entrevistador a otro, pensamos que no debiera imponerse esta tarea al equipo de salud.

Por otra parte, cuando nos cuestionamos la observación del principio de confidencialidad y el secreto profesional versus la entrega de información acerca de la concurrencia de los hechos constitutivos de una violación por parte del equipo médico, impresiona que esto conforma una franca contradicción excluyente respecto del rol médico y la función como testigo.

De esta manera el equipo psicosocial queda expuesto a un doble rol: por un lado, debe llevar a cabo la constitución de tercera causal, determinando si el relato de la paciente es o no plausible 
y si las fechas y hechos son o no concordantes con un embarazo producto de una violación, $y$, por otro lado, debe asumir el acompañamiento psicoterapéutico de la paciente. En este sentido, el equipo pudiese verse enfrentado al dilema de tener que hacer preguntas o aclaraciones en la entrevista que no cumplen una función terapéutica, $y$, por lo tanto, debiera estar muy consciente de no retraumatizar a alguien que ya sufrió un trauma grave reciente.

A modo de conclusión, es importante recalcar que la Ley IVE ha traído una oportunidad para que las pacientes que han sufrido un embarazo producto de una violación puedan, por un lado, decidir si quieren o no continuar con su embarazo $y$, por otro, tengan la posibilidad de recibir atención en salud mental por un equipo especializado. Sin quitarle mérito a lo anterior, hemos querido señalar algunos aspectos de la implementación de la Ley que han suscitado un cuestionamiento acerca de las implicancias de la participación del equipo psicosocial en la constitución de tercera causal y tensionan la relación terapéutica. Esperamos que los puntos planteados en este artículo puedan enriquecerse con la mirada de otros equipos IVE de nuestro país.

\section{Referencias}

1. United Nations, Department of Economic and Social Affairs, Population Division (2014). Abortion Policies and Reproductive Health around the World (United Nations publication, Sales No. E.14.XIII.11).

2. Ley No 21.030: Regula la despenalización de la interrupción voluntaria del embarazo en tres causales (23 de septiembre, 2017). Disponible en: https://www.leychile. $\mathrm{cl} /$ Navegar?idNorma $=1108237$ [Consultado el $12 \mathrm{de}$ julio de 2020].

3. Ministerio de Salud (Chile), Subsecretaría de Salud Pública. Norma técnica nacional - Acompañamiento y atención integral a la mujer que se encuentra en alguna de las tres causales que regula la Ley 21.030. Santiago; 2018. Disponible en: https://www.minsal.cl/wp-content/ uploads/2018/01/NORMA-ACOMPA\%c3\%91AMIENTO-Y-ATENCI\%c3\%93N-INTEGRAL-LEY-21.030.pdf [Consultado el 12 de julio de 2020].

4. Astete C, Beca JP, Lecaros U. Propuesta de un glosario para la discusión del aborto. Rev Med Chile 2014; 172: 1449-51.

5. Caro Miranda, J. Reflexiones "contractuales" acerca de la ley de interrupción voluntaria del embarazo en Chile. Acta Bioethica 2019; 25 (2): 215-24.

6. Bórquez G, Bernier L, Besio M, Montt J, Novoa F, Salas $\mathrm{S}$, et al. Reflexiones en torno al proyecto de ley (Boletín $\mathrm{N}^{\circ}$ 9895-11) que Regula la Despenalización de la Interrupción Voluntaria del Embarazo en Tres Causales. Rev. Chil. Obstet. Ginecol. 2015; 80 (3): 199-207.

7. Ministerio de Salud. (Chile), Subsecretaría de Salud Pública. Orientaciones técnicas - Acogida y acompañamiento psicosocial en el marco de la Ley 21.030, que despenaliza la Interrupción voluntaria del embarazo en tres causales. Santiago; 2018. Disponible en: https:// www.minsal.cl/wp-content/uploads/2018/03/OT-Acompan\%CC\%83amiento-Psicosocial-IVE-Resoluci\%C3\%B3n-Exenta-401.pdf [Consultado el 12 de julio de 2020].

8. Ministerio de Justicia (Chile), Servicio Médico Legal. Guía Normativa Técnica Pericial de Salud Mental para Pericias en las Áreas de Psiquiatría, Psicología y Trabajo Social Médico Legal. (8 de septiembre, 2015). Disponible en: https://www.leychile.cl/Navegar?idNorma=1081307\&idVersion=2015-09-08 [Consultado el 12 de julio de 2020].

9. Bergallo P, González Vélez A. Interrupción legal del embarazo por la causal violación: enfoques de salud y jurídico. Bogotá, La Mesa por la Vida y la Salud de las Mujeres (Colombia), Alianza Nacional por el Derecho a Decidir/ANDAR (México), Consorcio Latinoamericano Contra el Aborto Inseguro/CLACAI y Federación Latinoamericana de Sociedades de Ginecología y Obstetricia/FLASOG 2012.

10. Campohermoso Rodríguez O, Solíz Solíz R. Aborto terapéutico en Bolivia. Cuad. - Hosp. Clín. [Internet]. 2016; 57 (1): 72-82.

11. Stifani BM, Gil Urbano L, González Velez AC, Villarreal Velásquez C. Abortion as a human right: The struggle to implement the abortion law in Colombia. Int J Gynaecol Obstet. 2018; 143 Suppl 4: 12-8.

12. Diniz D, Madeiro A, Rosas C. Conscientious objection, barriers, and abortion in the case of rape: a study among physicians in Brazil. Reprod Health Matters. 2014; 22 (43): 141-8.

13. Ministerio de Salud de la Nación (Argentina). Protocolo para la atención integral de las personas con derecho a la interrupción legal del embarazo. Edición 2019. Disponible en: http://iah.salud.gob.ar/doc/433.pdf [Consultado el 16 de diciembre de 2020].

14. Corte Suprema de Justicia de la Nación (Argentina): Caso "F.A.L. s/medida autosatisfactiva", expediente 259/2010, tomo 46, letra F, sentencia del 13/3/2012. Disponible en: http://www.saij.gob.ar/corte-suprema-justi- 
cia-nacion-federal-ciudad-autonoma-buenos-aires--medida-autosatisfactiva-fa12000021-2012-03-13/1234567 89-120-0002-1ots-eupmocsollaf [Consultado el 16 de diciembre de 2020].

15. Black MC, Basile KC, Breiding MJ, Smith SG, Walters
ML, Merrick MT, et al. The National Intimate Partner and Sexual Violence Survey: 2010 Summary Report. (Atlanta, GA): National Center for Injury Prevention and Control, Centers for Disease Control and Prevention; 2011.

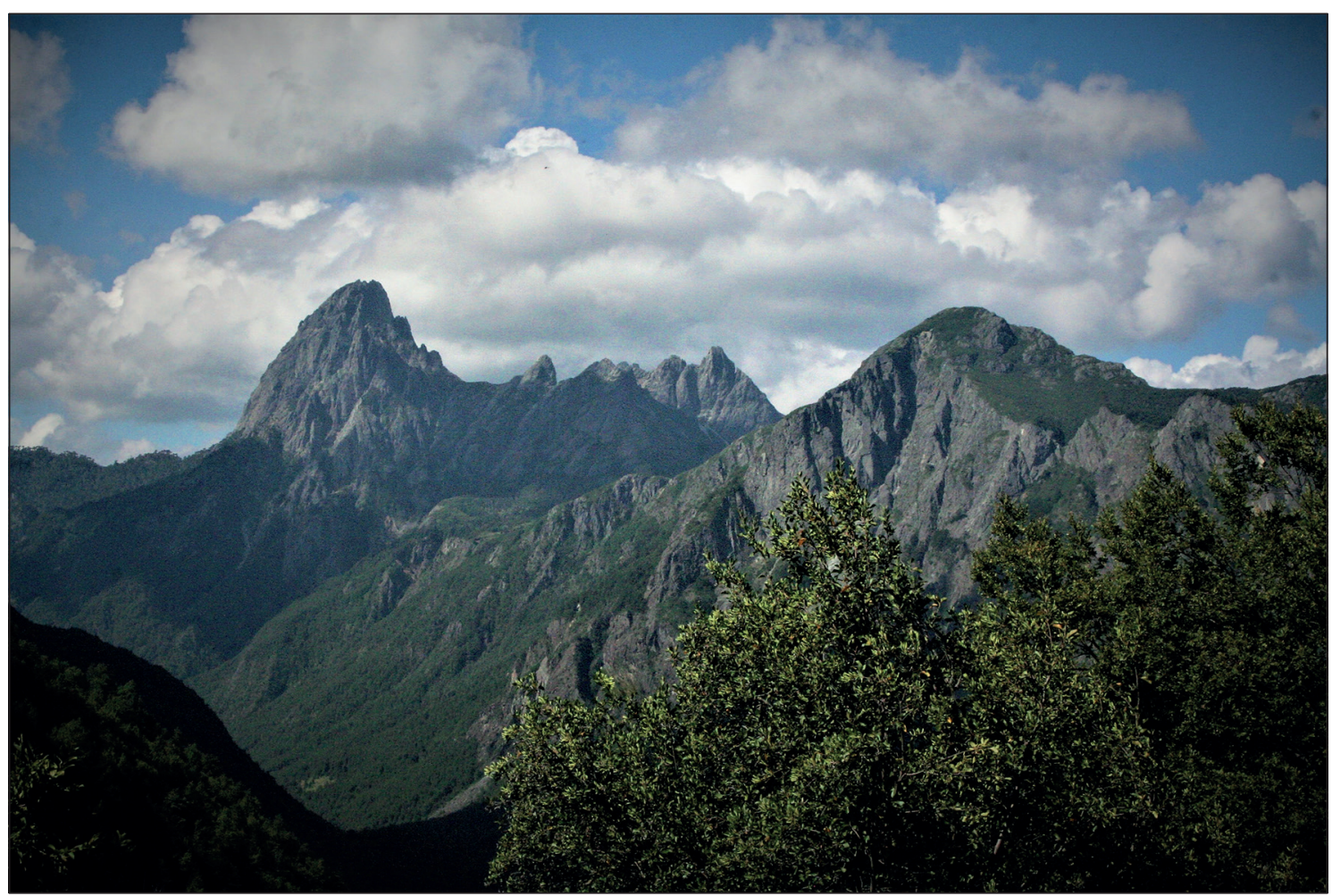

Sendero Laguna Avutardas, Curarrehue, La Araucanía. Dr. Jorge Sapunar Zenteno 